\title{
EchoGéo
}

$7 \mid 2008$

Les nouveaux enjeux régionaux dans l'océan Indien occidental

\section{Regards sur le Mozambique contemporain}

Dynamiques historiques et recompositions socio-spatiales d'une façade stratégique

\section{Fabrice Folio}

\section{OpenEdition}

\section{Journals}

Édition électronique

URL : https://journals.openedition.org/echogeo/8313

DOI : 10.4000/echogeo.8313

ISSN : 1963-1197

Éditeur

Pôle de recherche pour l'organisation et la diffusion de l'information géographique (CNRS UMR 8586)

Référence électronique

Fabrice Folio, «Regards sur le Mozambique contemporain », EchoGéo [En ligne], 7 | 2008, mis en ligne le 02 décembre 2008, consulté le 01 août 2021. URL : http://journals.openedition.org/echogeo/8313 ; DOI : https://doi.org/10.4000/echogeo.8313

Ce document a été généré automatiquement le 1 août 2021.

EchoGéo est mis à disposition selon les termes de la licence Creative Commons Attribution - Pas d'Utilisation Commerciale - Pas de Modification 4.0 International (CC BY-NC-ND) 


\section{Regards sur le Mozambique contemporain}

Dynamiques historiques et recompositions socio-spatiales d'une façade stratégique

\section{Fabrice Folio}

\section{NOTE DE L'ÉDITEUR}

Toutes les illustrations (imagettes) de cet article sont issues de « Exposiçao Moçambique : Vida e Historia em Psikhelekedana, » 1-19 juillet 2003, Centro Cultural Franco-

Moçambicano, Maputo ; photographie de Fatima Ribeiro, CIEDIMA. Elles sont toutes en rapport avec le tableau 1 (voir annexes).

1 Au sortir de plusieurs décennies de troubles, la république du Mozambique a recouvré une relative stabilité. Elle n'est certes pas le seul pays d'Afrique australe à avoir tardivement emprunté les chemins sinueux de la pacification, de la démocratie et de l'intégration à l'économie mondiale. L'exemple du voisin sud-africain est sans doute plus fréquemment énoncé en raison de la puissance médiatique qu'a revêtu, dans les décennies 80 et 90 , la lutte contre le régime d'apartheid. Mais rendre compte de cet Etat, aujourd'hui courtisé par les acteurs internationaux, nouveau champion de l'aide au développement et hôte de multiples investissements régionaux, mérite une attention particulière.

2 L'histoire tourmentée du Mozambique reste assez largement marquée du sceau d'enjeux extérieurs (Jouanneau, 1995). Ceux-ci ne se sont souvent que peu accommodés $\mathrm{du}$ sort des populations locales ${ }^{1}$. Traînant ce lourd héritage qui a pu freiner son développement, le Mozambique et en particulier sa capitale, Maputo, redevient un espace sur lequel il faut compter dans cette partie du continent. S'interroger sur ce qui explique ce renouveau et se pencher sur l'état des lieux de ce pays nécessitent deux préalables. En premier lieu, il convient de ne pas se focaliser uniquement sur le présent : le chemin qu'a parcouru le pays doit se mesurer à l'aune des louvoiements de 
l'histoire; des faits précis éclairent en effet la réalité spatiale contemporaine qu'une analyse géographique tentera d'expliciter. En second lieu, par sa position particulière et son état de primauté, il importe d'adhérer au jeu des échelles et d'analyser à sa juste valeur la région sud autour de Maputo; plaque tournante du territoire, elle est l'illustration même des nouveaux enjeux à l'oeuvre. En quoi l'organisation spatiale du Mozambique est-elle à la fois liée à des bifurcations historiques, aux ressorts souvent externes, et à des recompositions en cours, clairement ancrées dans la mondialisation ?

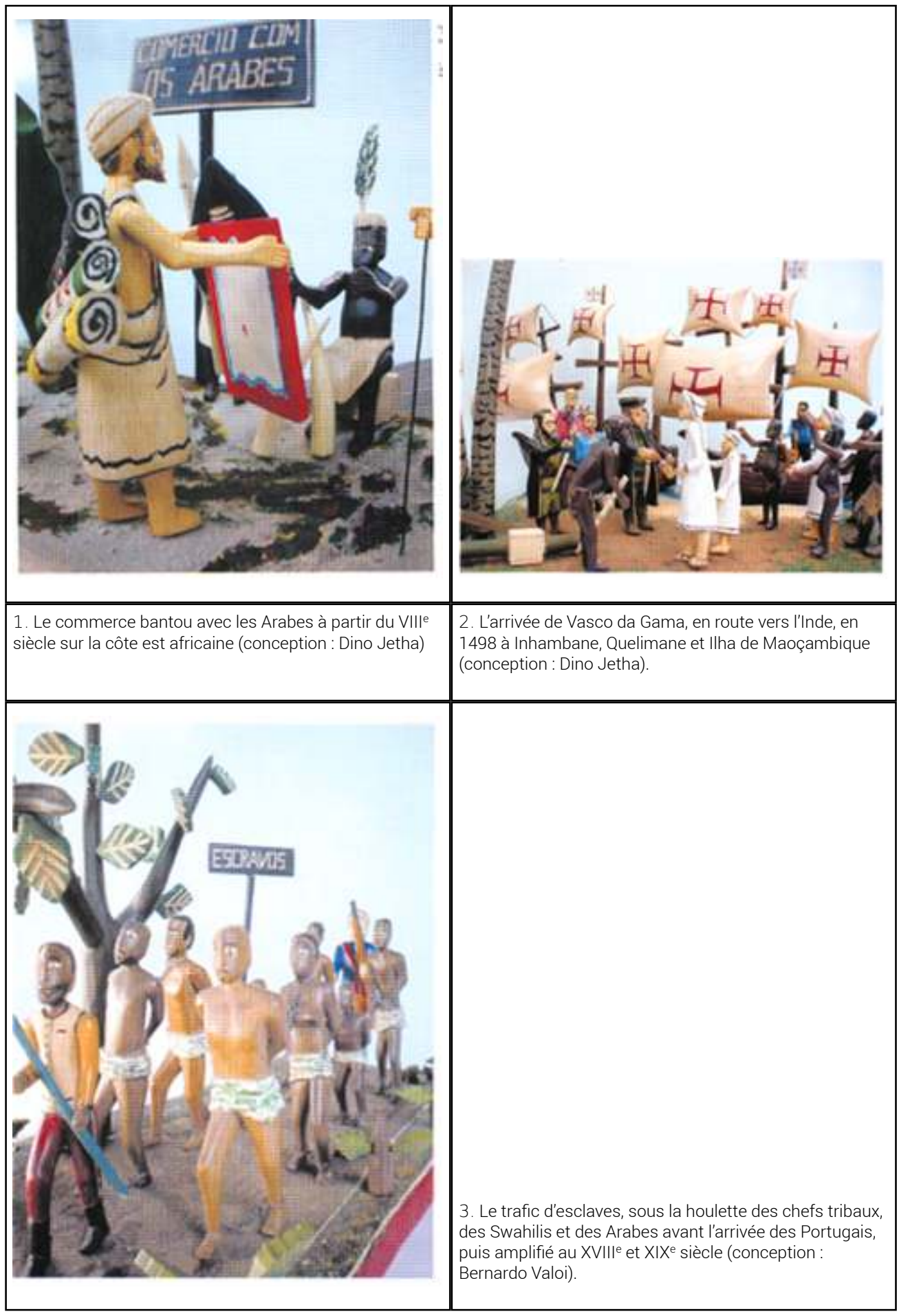


Cet article a pour but de porter un regard assez large sur les lignes de force - et leurs répercussions spatiales - du Mozambique. Tout en suivant ce fil directeur, nous tenterons de faire ressortir quelques traits de particularité du pays, lesquels soustendent l'idée de départ. Ainsi en est-il de cette forme singulière des frontières nationales. De même, à quoi peut-on imputer sur place cette contradiction apparente entre les indicateurs de croissance et ceux de développement? Enfin, peut-on parler d'une organisation spatiale axée, outre la primatie controversée de Maputo, sur des pénétrantes extraterritoriales et de nouveaux pré-carrés mondialisés ? Le texte s'ouvre sur quelques grandes observations qui structurent ce territoire. Il se penche ensuite sur la polarisation méridionale autour de Maputo, avant finalement d'esquisser l'organisation spatiale nationale, fruit des héritages et des mutations actuelles.

\section{Traits caractéristiques de ce pays et éléments d'explication géohistoriques}

Un certain nombre de remarques, qu'elles soient d'ordre territorial, socio-économique ou liées à l'aménagement de ce pays, peuvent être soulevées à l'observation du Mozambique.

5 Le pays s'allonge sur la côte est subsaharienne entre $10^{\circ} 27$ et $26^{\circ} 52$ de latitude sud et $30^{\circ} 12$ et $40^{\circ} 51$ de longitude est. De prime abord, le Mozambique présente une physionomie pour le moins originale. Un simple regard sur le tracé de ses frontières renseigne sur un trait de côte très étendu du nord au sud. Le pays ne s'élargit qu'aux confins nord et le long de la vallée du Zambèze et s'affine ensuite, au sud, en un mince liseré de terre. Le Mozambique affiche par conséquent une première singularité d'ordre géographique : son profil oblong, ou plutôt en "entonnoir ». Ses $2770 \mathrm{~km}$ de façade littorale s'étirent le long de l'océan Indien constituant la troisième surface littorale d'Afrique. Différents fleuves au débit important (Limpopo, Save, Zambèze, Lurio, Rovuma), à écoulement ouest-est, sectionnent cette écharpe territoriale et distinguent autant de régions qui restent mal reliées entre elles. Pour quelques pays enclavés (Zambie, Zimbabwe, Malawi), le Mozambique fait office d'interface maritime. Au total, le pays détient une frontière commune avec six Etats : dans l'ordre de grandeur, il s'agit du Malawi (1 $569 \mathrm{~km})$, du Zimbabwe (1 $231 \mathrm{~km})$, de la Tanzanie (756 km), de l'Afrique du Sud (491 km), de la Zambie (419 km) et enfin du Swaziland (105 km).

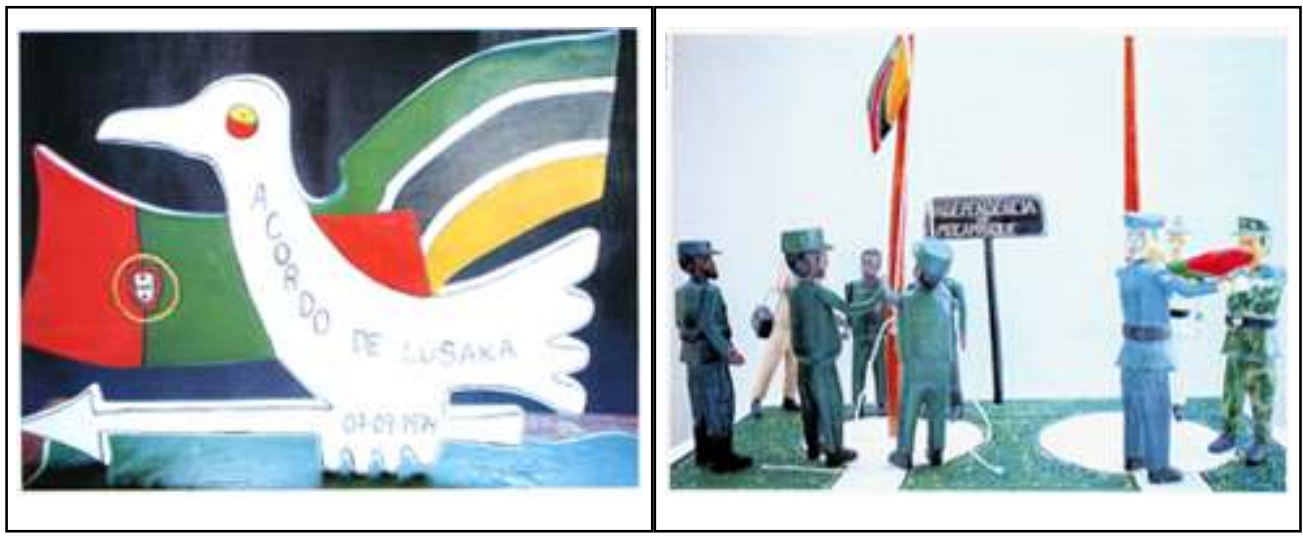


4. Les accords de Lusaka, signés le 7 septembre 1974, sous la pression de la lutte armée de libération, de

l'opposition interne et de la communauté internationale,

entérinant le droit du Mozambique à l'indépendance

(conception : Crimildo Cumbe).
5. L'indépendance du Mozambique le 25 juin 1975 et la fin des lois coloniales portugaises (conception : Dino Jetha).

Par ailleurs, les données préliminaires du recensement de 2007, disponibles auprès de l'Instituto Nacional de Estatistica (INE), indiquent une population totale de 20069738 habitants (soit un taux d'accroissement annuel moyen de 2,47\% depuis 1997). Un pourcentage significatif de cette population mozambicaine (plus de $48 \%$ ) vit dans les districts côtiers donnant sur le canal du Mozambique. En conséquence, les densités humaines restent en cet endroit les plus élevées (plus de $40 \mathrm{hab} / \mathrm{km}^{2}$ ), la densité nationale s'établissant pour sa part à $25,7 \mathrm{hab} . / \mathrm{km}^{2}$. Parmi les plus fortes occupations humaines relevées, il faut avancer les villes (cidades) littorales : outre les cités balnéaires (Xai Xai, Inhambane, Pemba) ou la localité historique de Ilha de Moçambique, citons la capitale Maputo (où sont recensés près d'1,8 millions d'habitants en 2007 pour une densité de $3664 \mathrm{hab} / \mathrm{km}^{2}$ ), située dans sa province éponyme au sud du territoire, Beira (436 240 habitants), la seconde ville au débouché du corridor de Sofala dans le centre et enfin Nacala (151 766 habitants), au delà du Zambèze, dans la province septentrionale de Nampula (figure 1). En d'autres termes, la façade côtière mozambicaine reste marquée par un chapelet lâche de centres urbains, pour beaucoup hérités des époques coloniale et précoloniale. Or, l'on sait que les villes précédemment citées sont aussi et avant tout des centres portuaires qui constituent la conclusion d'axes ferroviaires allant loin vers l'intérieur des terres, jusqu'aux pays riverains (Tanzanie, Malawi, Zimbabwe, Swaziland et Afrique du Sud). On saisit que l'organisation spatiale du Mozambique s'articule autour de «couloirs » parallèles qui, du nord vers le sud, composent autant de pénétrantes latitudinales. Sur ces axes estouest, se repèrent en effet quelques unes des principales villes de l'intérieur des terres, telles Chokwé depuis Maputo, Chimoio ou Tete depuis Beira ou encore Nampula et Lichinga depuis Nacala. 
Figure 1 - Carte du mozambique

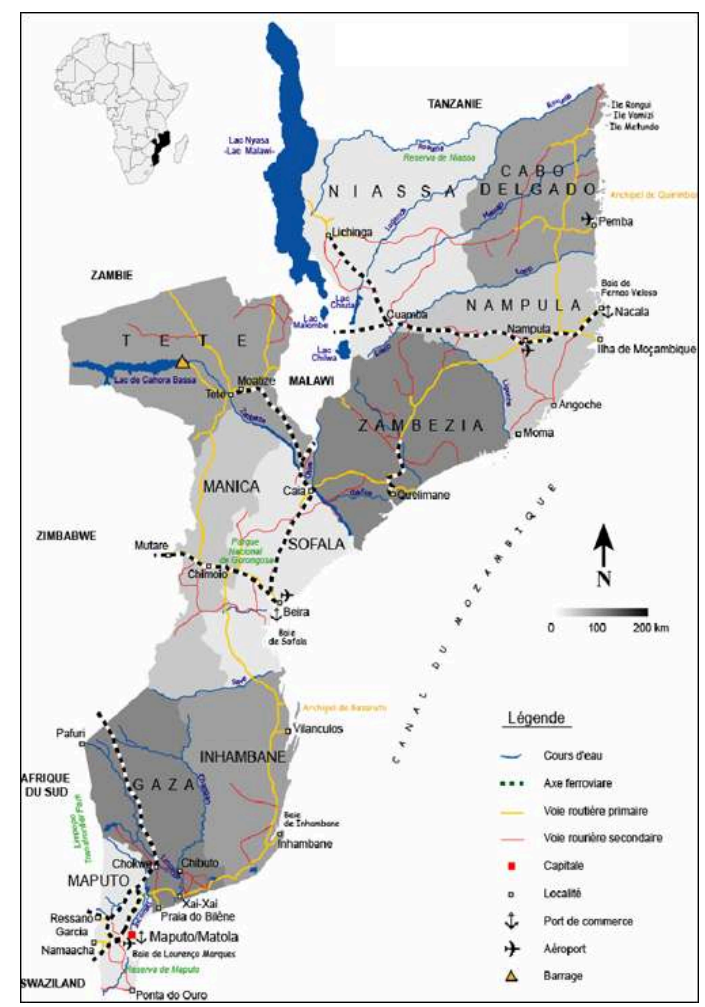

7 Troisième aspect intéressant de ce pays : son profil socio-économique. Il associe sans doute le pire et le meilleur sur l'échiquier mondial. Le Mozambique demeure l'un des pays les plus pauvres d'Afrique. Son produit intérieur brut est de 1237 dollars par habitant/PPA en 2004, ce qui le situe au $157^{\mathrm{e}}$ rang mondial sur les 177 pays listés par la Banque mondiale. Le rapport du PNUD sur le développement humain le positionne en 2006 en $168^{\mathrm{e}}$ position (sur 177 pays classés). Fixé à 0,40 USD par personne et par jour (BafD/OCDE, 2002), le seuil de pauvreté place $54 \%$ de la population en deçà (ce qui le met à distance des objectifs du Millénaire pour le développement). Présenté ainsi, le Mozambique reste clairement un PMA. L'annulation de sa dette auprès des institutions internationales le révèle, autant qu'elle constitue un premier pas attendu ${ }^{2}$. Le Mozambique reste de surcroît sous la menace continue de catastrophes naturelles, qui aggravent la situation de sous-développement (Foley, 2007). Parmi elles, on peut citer la sécheresse (en particulier dans le centre sud du pays, au climat tropical sec, avec une pluviométrie annuelle en deçà des 400 mm), les cyclones (Japhet en 2003, Favio en 2007) ainsi que les inondations. Ces dernières affectent tout particulièrement le centre du territoire. Elles sont dues aux pluies diluviennes et aux énormes quantités d'eau charriées par les fleuves. Outre les épisodes récents de 2007/2008 dans le bassin du Zambèze, on a tous en mémoire ceux de l'année 2000: ils ont causé la mort de 500 personnes, fait 300000 déplacés et mobilisé l'aide internationale. On aura été tout à fait complet en disant que le pays n'est pas à l'abri de critiques pour sa corruption importante (argent blanchi issu des trafics d'armes, de drogues ou du détournement de l'aide internationale), d'où son classement au $111^{\mathrm{e}}$ rang sur 179 pays en 2007, selon Transparency International. Prenant à revers ces remarques, le Mozambique se targue en parallèle d'offrir un modèle de redressement économique après un conflit armé. Le taux de croissance du PIB a été de $8 \%$ en moyenne sur la dernière décennie et de 7,9\% en 2006. Le pays est aujourd'hui courtisé par les investisseurs et les organismes de 
coopération, de même qu'il suscite une attention étroite de la part des organisations non gouvernementales (ONG). Fort d'une aide internationale massive (790 millions USD en 2004), le Mozambique mise sur la privatisation et les investissements directs étrangers (IDE) orientés vers l'exportation. Le cas de l'usine Mozal, à capitaux étrangers, située près de Maputo, est emblématique ${ }^{3}$. Au nombre des autres "mégaprojets" existants, on recense la mise en exploitation de champs gaziers à Inhambane par le groupe sud-africain Sasol, alors que l'initiative australo-sud-africaine Corridor Sands prévoit l'exploitation des gisements de titane des sables minéraux dans le sud du pays. En tout, à l'année 2005, le stock d'IDE totalisait 2,4 milliards de dollars ${ }^{4}$. Les trois principaux ports mozambicains, débouchés des corridors de Maputo, Beira et Nacala, ainsi que les ports dits secondaires (Quelimane et Pemba), ont fait (re)transiter 9,9 millions de tonnes de marchandises pendant l'année 2005 (CFM).

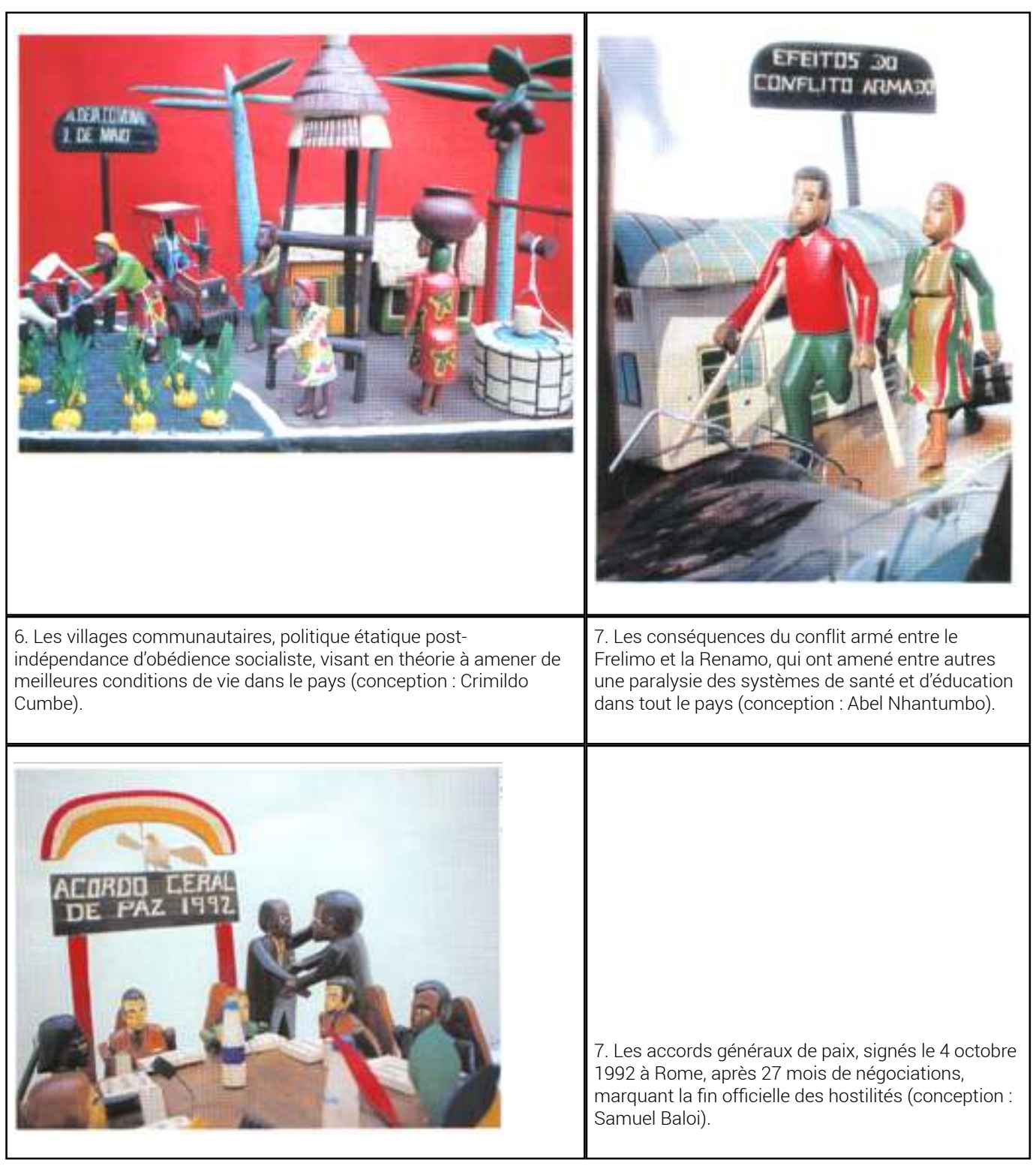

8 Ces éléments de singularité (forme géographique allongée, aménagement spatial en pénétrantes et pays pauvre à croissance économique dynamique), aux enjeux 
importants, trouvent un éclairage dans la lecture du passé. Pour le présenter, il convient au préalable de reconsidérer brièvement les trois phases historiques du Mozambique (pour plus de lisibilité et impression du tableau 1 et de la figure2 se reporter aux 2 annexes).

Tableau 1 - Les trois phases historiques du Mozambique (les numéros renvoient aux instantanés de la figure 2 qui se trouve en annexe)

\begin{tabular}{|c|c|}
\hline $\begin{array}{l}\text { Période } \\
\text { précoloniale } \\
\text { et coloniale }\end{array}$ & $\begin{array}{l}\text { Les commerçants arabes se sont longtemps affairés au négoce le long des côtes orientales africaines. Ils établirent, au nord de } \\
\text { l'actuel Mozzambique, plusieurs cités côtières (Sofala, Quelimane, Ilha de Moçambique) et ancrèrent ici une civilisation de type } \\
\text { swahili (1). Vasco de Gama aborde la côte mozambicaine en } 1498 \text { (à Inhambane et llha de Moçambique - 2). En 1507, une } \\
\text { période mercantile s'ouvre avec l'établissement de comptoirs portugais, le trafic d'ivoire et le début de la traite des esclaves } \\
\text { avec le Brésil en } 1645 \text { et les Mascareignes en } 1721 \text { (3). Le territoire est occupé par des prazos, soit un régime d'exploitation de } \\
\text { la terre et de ses habitants par un système de grands domaines concédés aux colons. En 1820, la colonie portugaise du } \\
\text { Mozambique devient une province outre-mer avec pour capitale llha de Moçambique. En 1891-1892, l'exploitation du pays } \\
\text { devient, pour } 50 \text { ans, le fait de capitaux étrangers (trois compagnies concessionnaires sont crées à cette fin). Après } 1926, \\
\text { année du coup d'état au Portugal, le genéral Salazar proclame l'Estado Novo. Le régime lisboète entend consolider la } \\
\text { bourgeoisie portugaise par le biais de la répression des travailleurs et du renforcement de l'exploitation coloniale. Sur le terrain, } \\
\text { est construit le barrage de Cahora Bassa. Les cultures de coton et de canne à sucre s'étendent. L'indigénat est institué, } \\
\text { réaffirmant le travail forcé. Au terme de plusieurs congrès du Front de Libération du Mozambique (ou FRELIMO), la lutte armée } \\
\text { débute en 1964, avant que la signature des accords de Lusaka, entre l'Etat portugais et le Frelimo, ne mène à un calme } \\
\text { précaire (4). A la suite de la révolution des ceillets au Portugal, la constitution d'un gouvernement de transition est entérinée en } \\
\text { 1974. }\end{array}$ \\
\hline $\begin{array}{l}\text { Période } \\
\text { post- } \\
\text { coloniale }\end{array}$ & $\begin{array}{l}\text { On parle aussi de période «socialiste », en vertu de l'indépendance acquise le } 25 \text { juin } 1975 \text {, date de la proclamation de la } \\
\text { république populaire du Mozambique (5). En } 1977 \text {, le Frelimo se transforme en parti marxiste-léniniste sous la houlette de } \\
\text { Samora Machel. Les administrations municipales coloniales sont dissoutes en } 1978 \text {, avant qu'une loi foncière (lei de terras), } \\
\text { promulguée en } 1979, \text { ne nationalise le foncier. La politique est axée sur la propriété collective des biens et des méthodes de } \\
\text { production, cependant qu'avec de maigres moyens consentis (financiers mais aussi en termes de formation et d'octroi de } \\
\text { compétences aux nouveaux cadres). L'accent est porté sur les campagnes avec la création de fermes d'Etat et de villages } \\
\text { communautaires de type Ujamaa tanzanien (6). En ville, les logements sont ouverts aux plus paurres. La ville socialiste restera } \\
\text { cependant ville du tiers-monde. En 1976, la guerre civile survient et s'étale sur plus d'une décennie (7). Elle oppose le Frelimo, } \\
\text { au pouvoir, aux rebelles mozambicains de la RENAMO (Resistência Nacional Moçambicana), groupe armé appuyé par des } \\
\text { intérêts extérieurs. Le pouvoir aux abois, de nouveaux modes de pensée se font jour au sein d'une géopolitique mondiale en } \\
\text { transformation. Les valeurs de l'économie libérale émergent. Le pays aborde timidement sa troisième phase, celle du } \\
\text { Mozambique en transition, ni socialiste, ni libéral, en crise et cherchant un soutien extérieur. }\end{array}$ \\
\hline $\begin{array}{l}\text { Période } \\
\text { actuelle }\end{array}$ & $\begin{array}{l}\text { Elle porte le sceau du changement à travers l'amorce démocratique et la vague néo-libérale. Ses racines se situent dans la } \\
\text { décennie } 80 \text {. En 1984, le pays adhéra à la convention de Lomé puis au Fonds Monétaire International (FMI). La même année } \\
\text { furent signés les Accords de Nkomati qui mirent fin à l'ingérence sud-africaine dans le conflit civil. En 1987, débutèrent les } \\
\text { politiques d'ajustement structurel (Programme de Réhabilitation Economique), à la suite de l'abandon progressif de la doctrine } \\
\text { marxiste-léniniste. La décennie suivante confirme cette tendance. En } 1990 \text { est promulguée la nouvelle constitution de la } \\
\text { république. En 1991, le Frelimo adopte le principe du multipartisme. En } 1992 \text { sont signés à Rome les accords de paix entre le } \\
\text { Frelimo et la Renamo (qui va se constituer en force politique d'opposition - 8). A l'année 1994, comme en Afrique du Sud, le } \\
\text { pays tient ses premières élections présidentielles et législatives au suffrage universel direct (9). Joaquim Chissano (Frelimo) est } \\
\text { élu président. Cette ultime phase se caractérise par la reconstruction (10), la stabilité et le multipartisme, mais aussi l'ouverture } \\
\text { tous azimuts: le Mozambique entérine une politique de libéralisation de son marché, soutenue par l'aide internationale. }\end{array}$ \\
\hline
\end{tabular}

Il semble que l'on puisse justifier les traits caractéristiques du pays en s'arrêtant sur quelques bifurcations historiques. Tout d'abord, s'agissant de la physionomie du territoire, elle trouve son explication dans l'ère coloniale. Présents sur le littoral depuis le XVI ${ }^{\mathrm{e}}$ siècle, les Portugais n'ont effectué une percée dans l'intérieur des terres qu'en suivant le Zambèze, vers l'illustre royaume de Mwene Mutapa et sa manne aurifère (Dictionnaire de Géopolitique, 1995). La situation fut loin d'être identique au nord et au sud. Fonctionnant de façon autonome, loin du support et de l'idéologie coloniale, les prazos centraux se sont fréquemment organisés en des domaines négriers. Le tout échappait au contrôle de Lisbonne : celle-ci ne détenait plus que quelques postes clés, où résidaient les colons (tels Ilha de Moçambique au nord ou Lourenço Marques au sud). Les compagnies concessionnaires, souscrites plus tardivement, ont confirmé cette délégation du pouvoir colonial dans la mise en valeur du territoire (de même qu'elles éclairent la gestion écartelée de la province). On était loin des objectifs initiaux lorsque, au faîte de leur impérialisme, les Portugais caressaient le rêve d'un empire rejoignant les deux rives du continent (soit leurs comptoirs d'Angola et du Mozambique). Or, depuis la fin du XIX ${ }^{e}$ siècle, le jeu de pouvoir avait changé dans cette partie du monde. Ils ne pouvaient plus guère donner le change à l'appétit des Britanniques et à leur dessein d'une liaison rejoignant Le Caire depuis Le Cap. Si en Angola on leur concéda une certaine liberté d'action - car l'arrière-pays était aussi d'un intérêt plus modeste -, une telle façade orientale ne leur permit pas d'agir ici selon leur volonté. En vertu de 
l'accord bilatéral de 1891 fixant leurs frontières, aucune concession ne leur fut faite, exceptée la zone du Zambèze. Dépendant de son mentor économique britannique, le Portugal ne fut finalement accepté qu'en tant que mandataire d'exutoires maritimes d'un riche hinterland. La province outre-mer devint avant tout un espace-débouché stratégique.

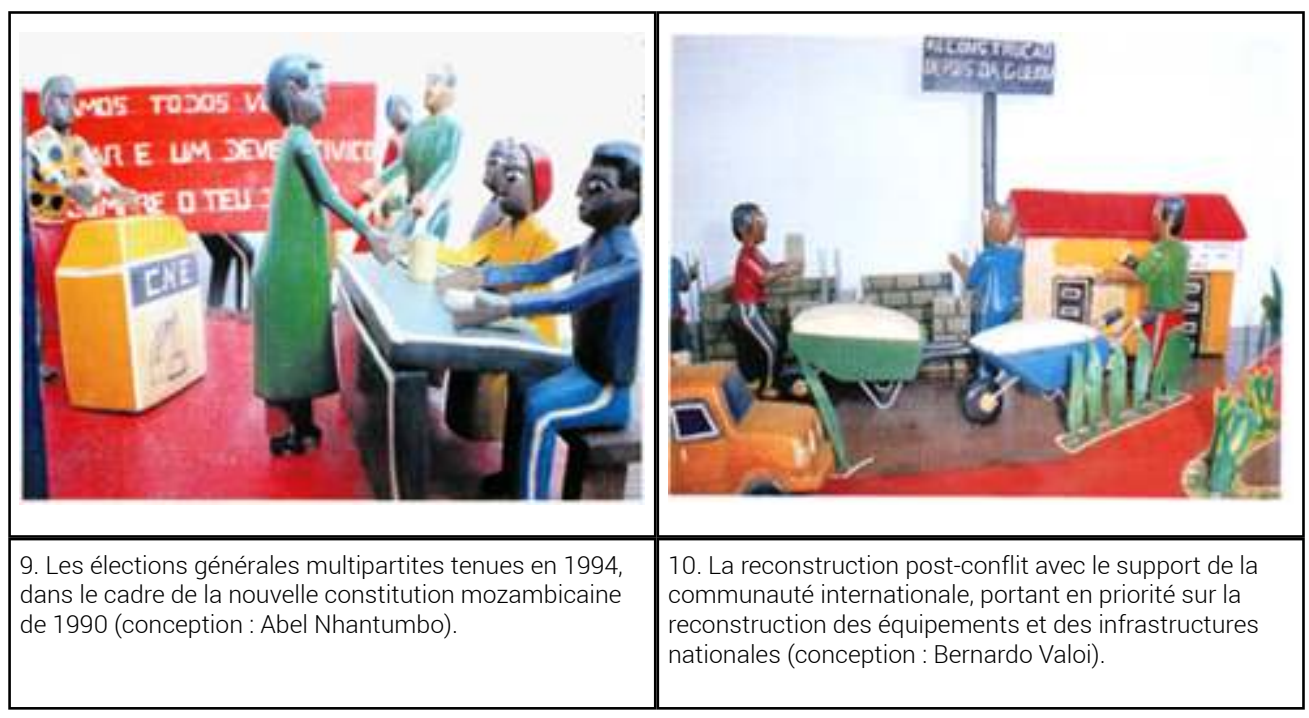

Ce point d'analyse est déterminant car il justifie également l'organisation du pays en corridors parallèles. Par là, on comprend que ces derniers restent mal reliés entre eux. A ce titre, on s'est souvent interrogé sur l'homogénéité du Mozambique. Les liaisons nord-sud ont toujours été dérisoires car négligées. Par exemple, une liaison par bac sur le Zambèze est actuellement nécessaire pour faire la jonction par route entre le nord du pays et le sud à concentration industrielle et commerciale. Lorsque l'on se penche sur ce découpage latitudinal, on constate que ce sont les voies ferrées qui en incarnent l'élément fort (en plus des obstacles naturels qu'incarnent les fleuves). Pour autant, parler de réseau ferroviaire est très contestable. Sur un total de $3000 \mathrm{~km}$, plus des deux tiers du chemin de fer du pays ont une utilité internationale. Du nord vers le sud, on relève successivement l'axe joignant le Malawi depuis Nacala, la voie qui relie ce pays au port de Beira, la ligne desservant le Zimbabwe depuis Beira, et enfin l'écheveau ferroviaire qui se noue à Maputo : à ce niveau, on dénombre une seconde ligne vers le Zimbabwe, le long du fleuve Limpopo, l'axe qui dessert le cœur économique sudafricain, le Gauteng (chemin de fer du Rand) et la ligne Maputo-Mbabane au Swaziland. Les ports ont donc un arrière-pays international (Guebourg, Brunet, 1997). Les villes littorales (notamment Maputo et Beira) représentent les terminaisons de ces axes historiques depuis les ex-empires coloniaux périphériques - notamment les possessions britanniques -, au potentiel minier indéniable. Ce sont les synapses d'un véritable système d'exploitation économique régional ${ }^{5}$. Ces enjeux ont été de tout temps supérieurs à toute politique d'aménagement intérieur. Au terme d'une période de crise liée au conflit civil, les ports mozambicains se positionnent à nouveau comme des fenêtres maritimes pour les pays proches. Le Mozambique est redevenu une plaque tournante dans le commerce régional ${ }^{6}$.

Pour terminer et dans le but d'appréhender cette dualité développement/croissance que mettent en lumière les indicateurs statistiques, il nous faut faire appel à deux 
bifurcations assez récentes. Les politiques d'ajustement structurel impulsées par le FMI en 1987 ont assaini l'état économique du pays. Toutefois, et à l'instar de ce qui s'est passé ailleurs, la population civile en a subi les contrecoups. Le pouvoir d'achat a accusé un brusque recul du fait des dévaluations du Metical (Mts). Les privatisations successives ont débouché sur des pertes d'emplois massives. Ces politiques structurelles se greffaient sur un pays qui avait subi le joug colonial et pâti de plusieurs années de guerre civile. En dépit de ces aspects, le cadre démocratique et libéral va stabiliser la situation intérieure et rendre le pays fréquentable sur la scène mondiale. En somme, il faut, d'une part, considérer l'état de délitement du Mozambique et la voie de la mondialisation et de la démocratie entérinée; d'autre part, prendre la mesure d'un Etat dont la position redevient géostratégique (beaucoup de pays limitrophes - et donc une partie de l'Afrique australe - dépendant, on l'a vu, de la stabilité in situ). Au final, le Mozambique devient rapidement éligible à diverses aides auprès de 48 bailleurs de fonds bi et multilatéraux, dont 10 agences spécialisées des Nations-Unies. Cependant, selon Harrison (1999), cette instabilité passée, associée aux creusements des inégalités dans un contexte libéral, a conduit à de vives tensions et contradictions sociales. Elles créent sur place un terreau aux pratiques corruptives. On peut dés lors s'interroger sur la confiance presque indéfectible placée en ce pays par les donneurs. Pour Hanlon (2004), cela s'expliquerait par le fait que certains donneurs ont besoin d'atteindre leurs objectifs internationaux en matière de coopération nord/sud et sont soumis aux pressions budgétaires émanant de leur gouvernement respectif. Aussi se satisfont-ils parfois de leur " bon élève » au sud, n'assurant qu'assez peu le suivi de la concrétisation des aides sur le terrain (ce qui n'exonère nullement les responsabilités étatiques locales). Aujourd'hui, l'aide étrangère finance $40 \%$ des dépenses publiques nationales. Le pays fait les yeux doux aux investisseurs (via une fiscalité favorable), au moment même où l'Afrique du Sud reprend sa place de géant sur le continent.

\section{L'extrême sud autour de Maputo : un espace primatial distant et sous influence}

12 Au-delà de ces observations initiales, la république du Mozambique reste surtout marquée par une autre réalité spatiale sur laquelle il nous faut nous attarder: l'excentration géographique de sa capitale, Maputo, et le sentiment d'un clivage apparent entre la première ville et le reste du pays. Pour l'illustrer, une des métaphores qu'utilisent fréquemment les humanitaires dans leur description du Mozambique, est celle d'un «sac », au fond duquel se tassent les deniers des projets internationaux et de l'aide multinationale. Or, ce clivage, très controversé, est assez curieux car il échappe aux grandes réalités naturelles et historiques du pays : la région centre-sud, en arrière de Maputo, présente en effet des conditions climatiques difficiles et reste moins peuplée à l'échelle nationale.

Cette dichotomie est déjà visible au plan humain avant le tournant des années 2000. Le poids de l'agglomération ressort alors de façon sensible. En 1997, du haut de ses 1391499 habitants, la première ville rassemble en effet 30,2 \% de la population urbaine nationale et 8,6\% de la population totale (en lui additionnant les chiffres de Matola, sa banlieue industrielle, ce qui constitue alors le "grand Maputo»). Peut-on dés lors parler de macrocéphalie urbaine ? En 2007, les chiffres officiels concèdent 1,77 millions d'âmes dans l'aire urbaine contre plus de 430000 habitants à Beira (INE, 2007). En 
réalité, cette concentration est d'autant plus notable que l'ex-Lourenço Marques borde des provinces sud peu peuplées (Gaza, Inhambane), loin des fortes densités du Nord, qui restent le cœur historique du pays. Dans les contrées de Zambézia et de Nampula, on recense effectivement plus de 3,8 et 4 millions d'habitants respectivement (avec des densités moyennes de 37 et $50 \mathrm{hab} . / \mathrm{km}^{2}$ ), contre un peu plus d'1,2 millions de Mozambicains dans celles de Gaza et d'Inhambane (où les densités s'abaissent à $17 \mathrm{hab} . / \mathrm{km}^{2}$ ). Plus largement, $61 \%$ de la population mozambicaine vit au-delà du Zambèze ( $76 \%$ si l'on prend pour limite la rivière Save). Aussi, plus qu'un écart rang/ taille conséquent (le ratio entre la capitale et la deuxième ville est de 4,1 ), c'est d'abord sa situation géographique "de confins sud" qui illustre la primauté de la capitale Maputo, loin du centre de gravité géographique national (figure 3).

La localité affiche en outre les indicateurs de développement les moins défavorables.

Figure 3 - Ville et densité de population au Mozambique

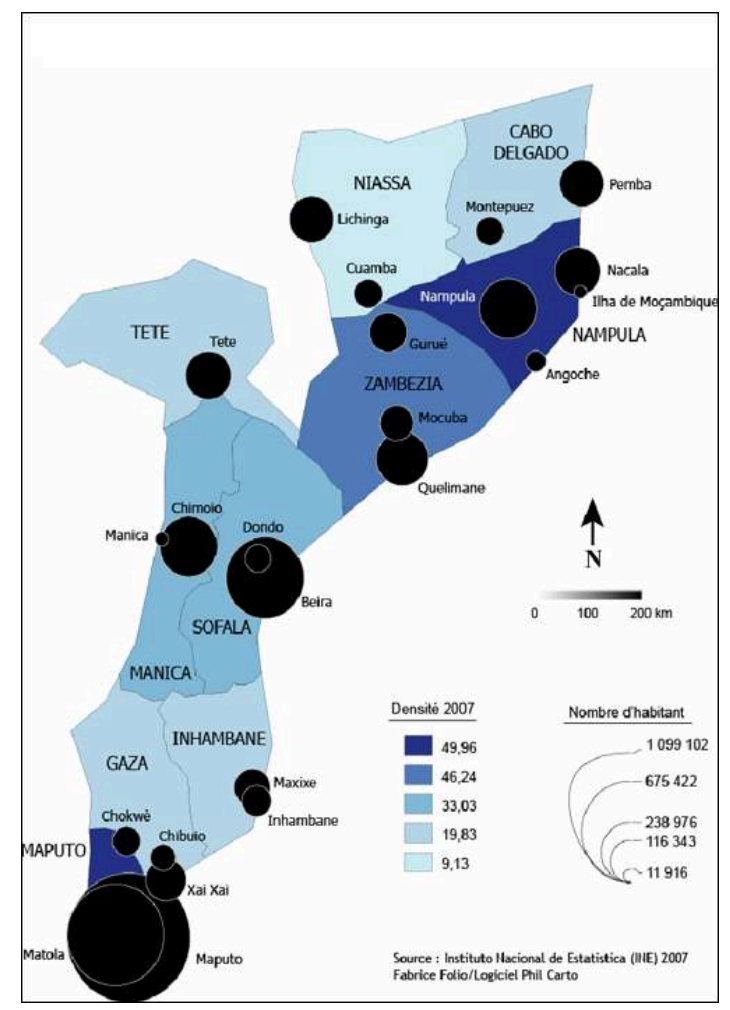

15 Les statistiques du PNUD à l'année 2001 révélaient déjà que les provinces centre et nord accusaient des valeurs infimes qui tranchaient avec celles de la province de Maputo et surtout avec celles de la capitale. Les données les plus récentes, publiées par le Southern African Research and Development Centre (SARDC) et financées par le PNUD, vont dans le même sens. L'IDH de la ville de Maputo en 2004 est de 0,651, celui de sa province de 0,588, tandis que les indicateurs les plus médiocres sont à chercher plus au nord : 0,353 à Niassa, 0,340 à Nampula ou encore 0,313 à Cabo Delgado. Un autre indicateur, très utilisé au Mozambique, est celui mesurant la pauvreté humaine (associant indicateur de longévité, d'instruction et de conditions de vie). Il atteste là encore d'une amplitude notable entre la capitale $(18,4 \%)$ et le reste du pays, où les taux, hors province de Maputo, s'établissement toujours au dessus des $35 \%$ (figure 4). Dans le même ordre d'idée, l'espérance de vie est de 52,1 ans à Maputo-ville contre 37,5 ans en Zambezie; le 
taux de mortalité infantile, de 178 décès pour 1000 naissances au Mozambique, oppose la capitale (89 pour 1000) à Cabo Delgado, où 241 enfants sur mille meurent avant l'âge de cinq ans (ESDM, 2004). Il est évident que ces chiffres font toutefois l'impasse sur les vives inégalités internes.

Figure 4 - Indicateur économique et de développement au Mozambique

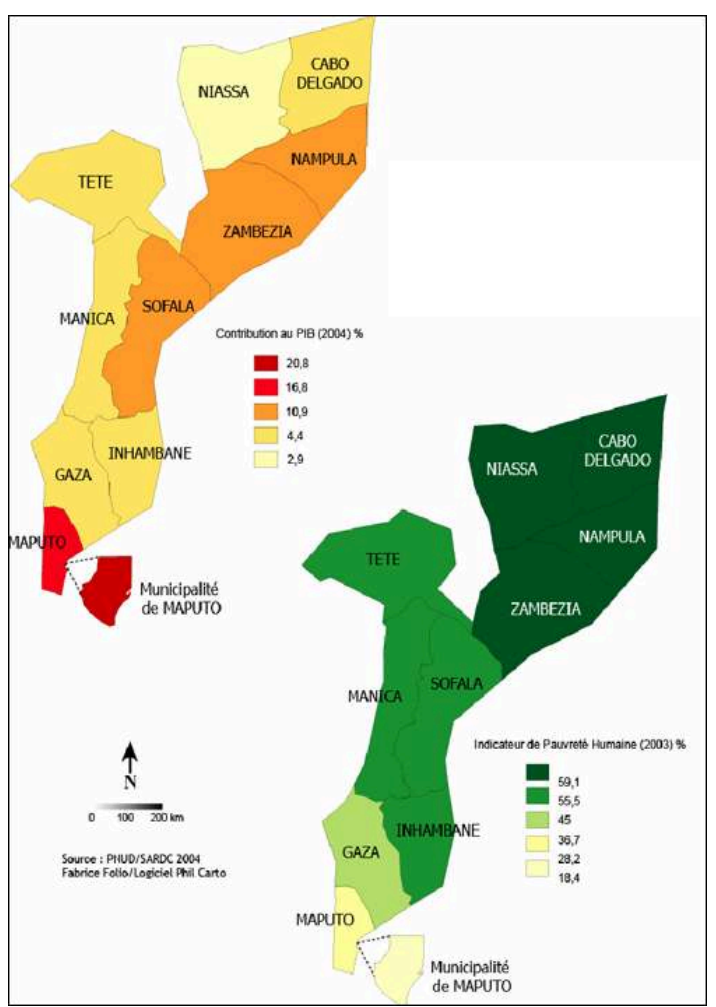

$16 \mathrm{Au}$ niveau économique, si l'on se réfère aux performances des provinces (figure 4), la contribution du sud du pays au produit intérieur brut national est incontestable : elle s'établit à $37,6 \%$ pour la seule province de Maputo, dont $20,8 \%$ pour la première ville (PNUD/SARDC, 2007). Cette dernière rassemble l'essentiel des industries nationales et de la fonction tertiaire inhérente à son statut de capitale. Mais, alors que les conditions climatiques restent plus humides au nord, sur la zone des plateaux fertiles de Nampula et de Cabo Delgado (dégageant parfois des surplus alimentaires, notamment de maïs), le jeu politique, qui se superpose aux faits historiques et à la carte ethnique, maintient ces espaces dans un relatif isolement, inhibant leur performance et leur intégration à l'économie nationale. Les infrastructures, les services comme le secteur privé y sont moins représentés que dans le sud. En 2005, par exemple, la crise alimentaire qui a frappé certains secteurs arides méridionaux a rendu très cher l'acheminent des denrées du nord vers le sud, en raison du mauvais état du réseau des transports. Tout cela n'en rend que plus sensibles les débats sur la scène politique avec la question de la réconciliation nationale en arrière-plan. Elle s'est par exemple cristallisée dans les urnes lors des premières élections libres de 1994 : une démarcation nord/sud a opposé les deux formations politiques en présence. La Renamo, à la surprise des observateurs, est arrivée seconde en rassemblant $38 \%$ des votants (contre $44 \%$ pour le Frelimo). Elle a remporté les provinces centrales et septentrionales, à l'exception notable de Cabo Delgado et de Zambezia (le Frelimo arrachant finalement Tete, Nampula et Niassa lors des élections de 2004). Pour comprendre cette réalité, pour le moins sensible sur le 
terrain, il convient encore d'opérer un retour à l'histoire et de cerner le contexte hérité dans lequel s'inscrit ce clivage.

En 1887, Lourenço Marques, du nom du commerçant portugais qui effectua une reconnaissance de la baie en 1544-45, est élevé au rang de ville. La ligne de chemin de fer vers Johannesburg, région alors en plein essor, est ouverte en 1895. Trois ans plus tard, la capitale du Mozambique est transférée du nord, d'Ilha de Moçambique, au sud à Lourenço Marques. La finalité de ce décrochement est la connexion de Lourenço Marques aux zones dynamiques de la Rhodésie du Sud et de l'Union sud-africaine, prétexte à l'établissement d'un débouché commercial. Depuis 1896 et les contrats passés avec la Witswatersrand Native Labour Association, le travail migrant consacrait aux mines sud-africaines des quotas de travailleurs africains. L'accord de 1901 entre le Portugal et le Transvaal permet le recrutement de mineurs dans l'espace mozambicain situé au sud de la rivière Save. Cette exportation de main d'œuvre va dans le sens des intérêts sud-africains comme portugais ${ }^{7}$. Les travailleurs remplissent à ce moment les hostels - foyers ouvriers masculins - des mines aurifères et charbonnières du Rand. Cette spécificité du travail migrant va graver une réalité migratoire anisotropique dans cette partie de l'Afrique australe 8 . A cette période, le port de Lourenço Marques connaît lui aussi un essor notable. En 1928, un accord est signé qui concède au port 47,5 \% du trafic maritime des zones du Rand. Le débouché mozambicain devient d'autant plus prisé que sa position joue pour lui (les ports sud-africains se situant à plus grande distance des mines des hautes terres). A cet effet, le trafic du port de Maputo atteindra les 12,5 millions de tonnes en 1969 (Engineeringnews, 2006). L'interdépendance avec les intérêts britanniques proches devient réelle tandis que Lourenço Marques se vante d'être devenue l'une des villes d'Afrique les plus élégantes... Au nord, les vieux noyaux créoles issus, entre autres, du trafic d'esclaves sont marginalisés. La mise en valeur du territoire reste ardue et coûteuse. Les compagnies à charte avaient globalement laissé des terres pauvres et mal contrôlées (Gervais-Lambony, 1997). La politique coloniale volontariste de Salazar ne peut inverser la tendance. A l'échelle du pays, la distinction socio-spatiale apparaît.

Lorsque survient la période de l'indépendance, la lutte pour la libération est conduite par un seul mouvement, sous la direction d'Eduardo Mondlane, proche du tanzanien Julius Nyerere. Toutefois, elle est menée sur deux fronts avec des modalités d'actions différentes. Au sud, autour de l'ethnie shangane principalement (qui appartient au peuple Tsonga), s'organise la direction du mouvement du Frente de Libertação de Moçambique. Le nord, pour sa part, est davantage l'épicentre de la lutte armée, s'exprimant avant tout autour des Makonde à Cabo Delgado (Le dictionnaire historique et géopolitique du $20^{e}$ siècle, 2005). Quant au centre et au centre nord du pays, ils ne sont que peu intéressés par ce mouvement d'émancipation. La guérilla ne s'y implante que très peu. Au terme du retrait brutal des Portugais en 1975 (conduisant à une saignée dans les postes à expertise), les dirigeants du parti prennent ancrage à Maputo. Conservant les réflexes d'une ère lusophone polarisée, adoptant les préceptes d'un régime marxiste-léniniste autocentré, l'appareil d'état du nouveau pays indépendant s'arrime clairement à la capitale. Il privilégie encore les ethnies sudistes, shangane et rhonga en tête (le soutien des combattants du nord tendra même à s'estomper auprès de la nouvelle élite). L'écart Maputo/reste du Mozambique s'accentue.

19 C'est dans ce contexte qu'émergent les bandes du Renamo. Ces dernières s'opposent à la politique d'obédience centralisée, bureaucratique et par trop méridionale du 
Frelimo. Notons que le conflit a souvent été présenté - par le Frelimo mais aussi par nombre de médias de l'époque - comme une entreprise de déstabilisation régionale et non un conflit civil. Il est avéré que la Renamo a été conçue à l'initiative de la Rhodésie du Sud puis soutenue par le parti national afrikaner. Toutefois, elle a bel et bien trouvé une assise dans les zones rurales mozambicaines (en particulier auprès des Ndau et Sena peuplant Manica et Sofala). Ces dernières ont fini par s'élever contre les villages communautaires (aldeias communais), imposés dans un monde de paysannerie traditionnelle à habitat dispersé. Les lignes de fracture ethniques créées par l'implication dans la traite (les Makonde face aux razzieurs macua et yao - Péllisier, 1987), de même que la très inégale complicité des communautés dans la lutte pour l'indépendance, sont aussi entrées en ligne de compte, quoi qu'ait pu dire le Frelimo.

De 1976 à 1992, le Mozambique sombre dans la guerre civile. Le pic de violence est atteint dans la seconde moitié de la décennie 1980. Au total, le conflit fera 1 million de morts, 6 millions de déplacés (hors du pays mais aussi à l'intérieur, les deslocados) en plus des attentats contre les infrastructures, avant tout en monde rural (Serra, 2003) ${ }^{10}$. Les soubresauts du conflit vont précipiter les masses humaines vers les villes côtières. Les populations s'y entassent ${ }^{11}$. Vers la fin des années 1980 , on prend la mesure de la crise urbaine au plan national (Lachartre, 1997). De 700000 âmes en 1970, on est passé à un total de 4604384 citadins en 1997. Le taux d'urbanisation, faible en 1970 (5,7\%), atteint $28,8 \%$ en 1997 . Alors que le pays connaît des taux de croissance annuelle de $1,6 \%$ (1980-1991) et 1,85 \% (1991-1997), le taux moyen en ville s'établit respectivement à 6,1 puis $7,6 \%$ sur les deux périodes. Le dynamisme de Maputo connaît alors un coup d'arrêt, même si la capitale n'est pas affectée directement par le conflit. Elle devient une ville dégradée en son centre, entourée de zones d'habitat précaire (les caniços). Réhabilitation, attractivité et croissance y sont en fait des réalités très récentes.

21 En résumé, l'actuelle primauté de Maputo s'inscrit dans un contexte libéral qu'il nous faut maintenant développer. Mais ses racines se situent dans les répercussions de la guerre, au sens ou Maputo fut un réceptacle pour les migrations interrégionales du sud du pays, parmi les plus intenses de toutes. Avant cela, l'ère socialiste avait déjà mené à une concentration méridionale dirigiste. Enfin, si l'on remonte encore le cours de l'histoire, il convient de ne pas occulter les conséquences coloniales qui se sont traduites par le déplacement de la capitale. Par certains égards (enjeux miniers, décrochage plus au sud), il rappelle le premier changement de capitale du Brésil en 1763 (de Salvador da Bahia à Rio de Janeiro).

\section{Une organisation spatiale qui se complexifie : entre héritages historiques et recompositions néo-libérales}

L'espace mozambicain fait écho aux trajectoires historiques dressées jusqu'ici. Mais il s'inscrit aussi dans des recompositions à l'œuvre, aux influences exogènes, inscrites dans la mondialisation. Celles-ci semblent s'absoudre d'une recherche de cohérence territoriale, soulevant par la même quelques questions dans les champs sociaux et environnementaux. L'ébauche d'organisation spatiale dressée ici est à rapprocher de la figure 1.

Parler de contrastes spatiaux au Mozambique, revient avant tout à faire ressortir la césure entre l'extrême sud, polarisé par la capitale, et le reste du pays. Il s'agit de la 
principale clé de lecture de l'organisation spatiale nationale et elle est de nature à se renforcer. La capitale est devenue la « vitrine » du nouveau Mozambique. Elle attire de nouveau les touristes en jouant de sa carte culturelle (patrimoine portugais, quartier et marché populaire) et balnéaire (Costa do Sol, île d'Inhaca). Ses facettes latine et décontractée séduisent. Les élites du parti au pouvoir, les coopérants ainsi que les entrepreneurs étrangers s'y sont établis (Lundin, 2000). Maputo a connu un vif essor économique ces dernières années, profitant de la nouvelle donne libérale et de sa proximité avec l'émergent voisin régional (avec qui les relations se sont normalisées). Les investisseurs sud-africains (plus de 250 sociétés présentes) y prennent ancrage (Daniel, Naidoo, Naidu, 2004). Un des symboles de la nouvelle réussite nationale est la remise sur pied des corridors de développement, en tant que débouchés naturels des pays frontaliers. Là encore, celui de Maputo en est le plus actif. A l'initiative du système BOT (Build, Operate and Transfer), il branche à nouveau le pays à l'économie sudafricaine ${ }^{12}$. Grâce au consortium TRAC (Trans African Concession, composé de compagnies locales et sud-africaines) et à la société portuaire et ferroviaire Companhia de Portos $e$ Caminhos de Ferro de Moçambique (CFM), il entend vivifier ce couloir de $503 \mathrm{~km}$ entre les deux pays, pour une meilleure intégration de la région. Déjà, le volume de trafic entre Witbank et Maputo s'élève à 60000 véhicules par jour en moyenne. Les visas ont été abolis. Afin de renforcer le rôle du secteur privé dans le port de Maputo, la gestion portuaire a été confiée à $51 \%$ à un consortium emmené, entre autres, par le britannique Mersey Docks Group. La réhabilitation en cours du port de Maputo lui permet aujourd'hui d'atteindre un trafic de 6,38 millions de tonnes. Ces projets sont soutenus par la South African Development Community (SADC) dans le cadre de ses initiatives régionales.

Cette polarisation méridionale est-elle vouée à s'accentuer? Les développements, en cours et à venir, semblent l'attester car ils jouent encore pour Maputo et la région côtière alentour. A l'échelle du pays, les futures entrées d'IDE devraient s'intensifier avec la mise en route de projets dans deux secteurs phares: le secteur minier et industriel et le tourisme. Dans le sud précisément, en plus du projet Corridor sands mené par BHP Biliton (aux environs de Maputo), une nouvelle expansion de l'usine Mozal (Mozal 3) devrait encore augmenter ses capacités de 250000 tonnes. Dans le domaine touristique, la création du parc transfrontalier du Limpopo, de concert avec l'Afrique du Sud et le Zimbabwe, revêt beaucoup d'enjeux. On peut émettre l'hypothèse que Maputo devienne une nouvelle base arrière pour la découverte de la réserve animalière, la plus grande du monde, abritant notamment l'illustre parc Kruger ${ }^{13}$. Dédié à la vie sauvage, le parc transfrontalier se situe en effet à plus faible distance de la capitale mozambicaine (moins de $100 \mathrm{~km}$ via la nouvelle route à péage de Ressano Garcia), que de l'aéroport de Johannesburg en Afrique du sud, la porte d'entrée du pays (située à $300 \mathrm{~km}$ ). D'autres aménagements touristiques existent ou émergent sur les marges nord (plages de Bilène) et sud de Maputo (réserve spéciale de Maputo, Ponta do Ouro au sein du Lubombo Spatial Development Initiative, programme de mise en valeur agricole et touristique associant l'Afrique du Sud et le Swaziland). Avec la réfection des routes, ils visent à structurer cet espace côtier et de l'intérieur proche des provinces de Maputo et de Gaza. Toute cette zone, à n'en pas douter, va enfin tirer profit de la proximité géographique liée à l'effet coupe du monde de football 2010. Le Mozambique compte en effet capter une partie des visiteurs. 


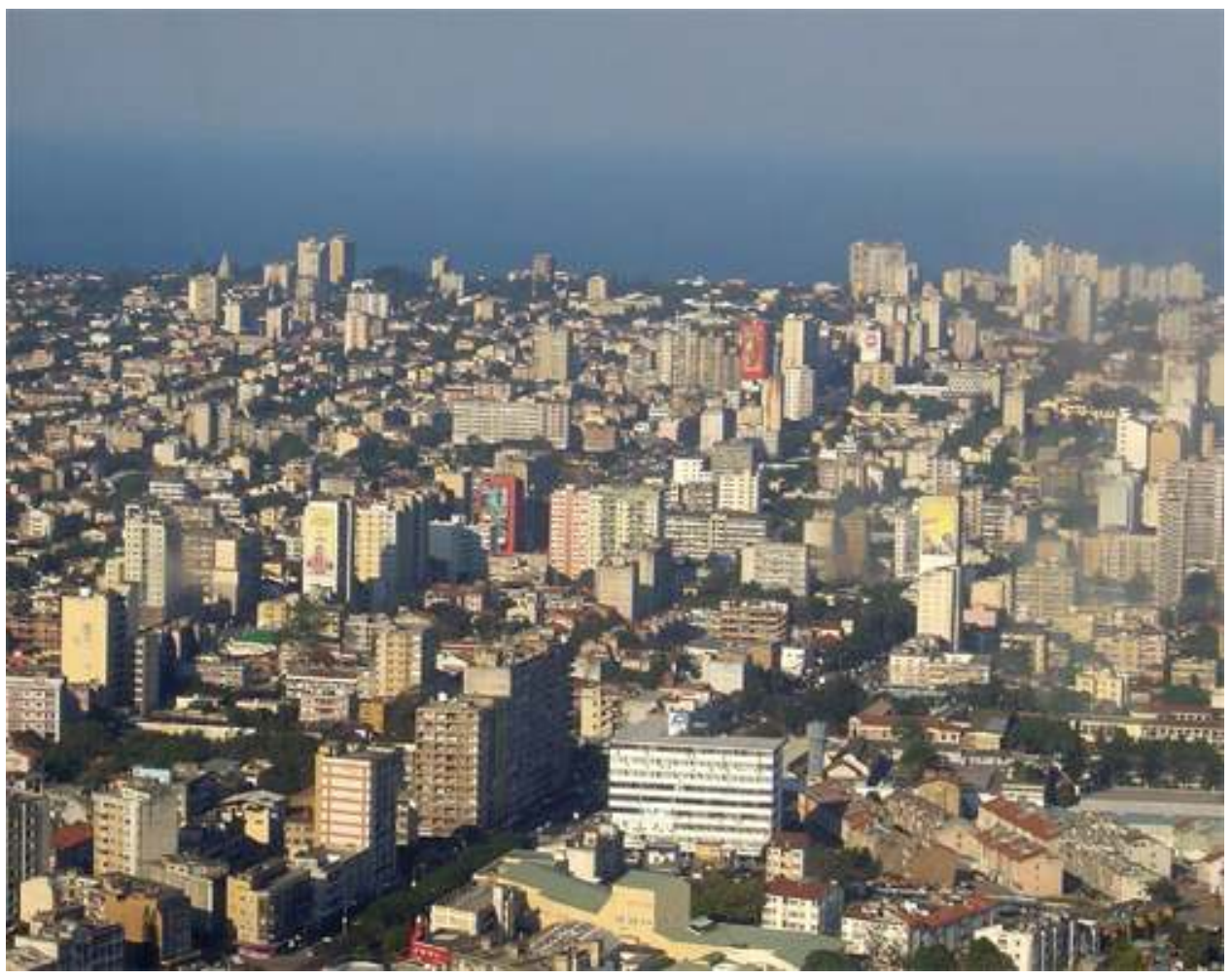

Figure 5 - Ci-dessus, la capitale, Maputo, en vue aérienne. La ville se compose d'une partie en dur, héritée de la colonisation, qu'enceingnent des espaces populaires d'habitat spontané. Ci-dessous, des habitations rurales, sur la route menant à Xai Xai, constituées en partie de matériaux végétaux(clichés : auteur).

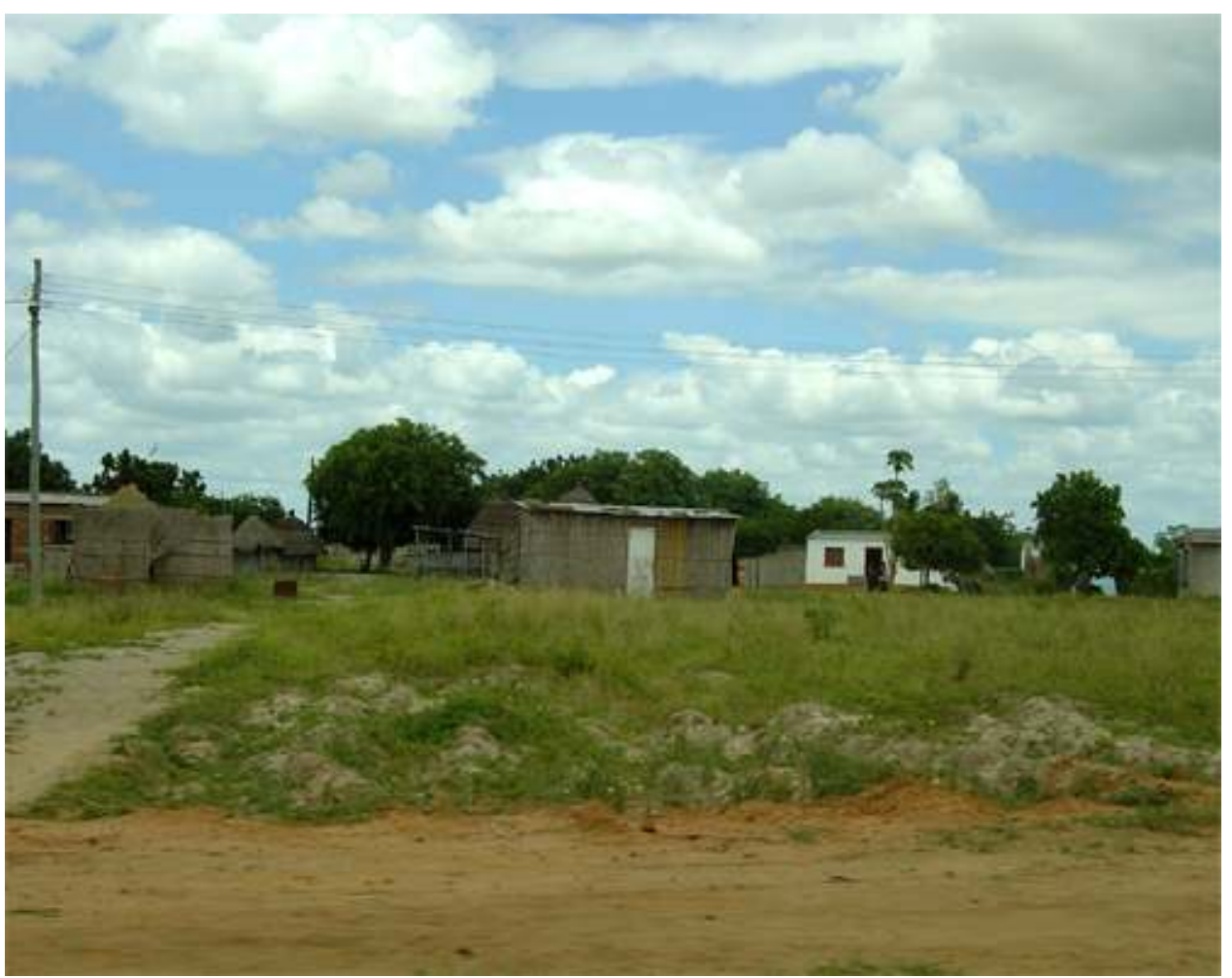

Dans le nord du pays, la situation, tout particulièrement en milieu rural, reste plus incertaine, ce que dénoncent périodiquement les cadres de la Renamo. Notons tout de 
même une réalité spatiale pesante qui se superpose au clivage sud/nord: l'opposition urbain/rural. Elle est prégnante au-delà du seul cas de Maputo. L'enquête IAF menée en 2002/2003 atteste d'un écart préoccupant dont témoignent plusieurs indicateurs de développement ${ }^{14}$. A cette double articulation (sud polarisé par Maputo/nord mal intégré et ville/campagne), il faut également cerner la place des corridors ouest-est. A ce niveau, on peut parler d'une certaine consolidation, dont devraient tirer profit les deux pôles de Beira et de Nacala. Effectivement, après les actes de sabotages qu'ils ont subis durant le conflit, on a pu craindre un déclin des corridors septentrionaux. Leur éloignement de Maputo et de l'Afrique du Sud, associé à l'instabilité régnant au Zimbabwe, joue en leur défaveur. Or, les initiatives récentes inspirent plus d'espoir. La reprise de l'exploitation des mines de charbon à Moatize, près de Tete dans l'intérieur, devrait donner un nouvel élan à la plate-forme de Beira avec la remise en état de la voie ferrée de Sena. Les travaux sont réalisés par un consortium indien: Rites and Ircon International. Quant au corridor de Nacala, sa fréquentation s'accroît du fait de l'évacuation du cuivre zambien (vers la Chine et l'Inde) et le développement de la voie ferrée et du port par la Société de Développement du Corridor de Nacala (SDCN), consortium américain, sud-africain et mozambicain. A Nacala, une raffinerie de pétrole, à capitaux texans, verra bientôt le jour au sein d'une zone franche industrielle, pour exportation vers les pays arrière.

26 Parallèlement, d'autres initiatives économiques, liées à des investissements étrangers, sont en cours bien que circonscrites spatialement. On peut à ce niveau parler de recompositions car elles vont probablement complexifier les lignes de force du pays décrites précédemment. En premier lieu, dans le domaine des activités extractives, le gigantesque projet d'extraction de matières premières du Moma Titanium au sud de Nampula est quasi achevé. A l'initiative de la société irlandaise Kenmare Ressources, le projet prévoit une extraction annuelle de 750000 tonnes de sables minéraux. Au nord toujours, au large du Rovuma Basin, des projets d'extraction off-shore de pétrolepourraient aussi se concrétiser (Bucuane, Mulder, 2007), les concessions ayant déjà été accordées à des compagnies étrangères (Petronas, Anadarko, Norsk Hydro...). En second lieu, il faut citer les développements touristiques dans le parc de Gorongosa (réintroduction d'animaux sauvages), à Inhambane et surtout dans les archipels de Bazaruto (près de Vilanculos) et de Quirimbas non loin de Pemba (à Cabo Delgado). Ces derniers restent les principaux pôles balnéaires du Mozambique rassemblant, hors de Maputo, la majorité des aménagements hôteliers de standing. Il est vrai que le climat tropical à fort ensoleillement, couplé aux fonds récifaux et à une eau india-océanique couleur turquoise, invite ici au tourisme dans un décor paradisiaque. Au sein des deux archipels, l'essor touristique souhaite s'adosser à la protection d'espaces sensibles ${ }^{15}$. A Quirimbas, le riche patrimoine de l'île d'Ibo répond aussi à celui de l'île du Mozambique près de Nacala. Les investisseurs étrangers accourent: outre le groupe portugais Pestana, la société émirati Rani investira 120 millions USD dans les trois prochaines années à Inhambane et à Cabo Delgado.

De nouveaux secteurs semblent ainsi émerger du territoire mozambicain dans une logique d'ouverture du marché. Toutefois on peut mettre deux bémols. D'une part, la façade littorale mozambicaine reste clairement avantagée. D'autre part, il semble s'agir d'un développement sous forme de pré-carrés voire "d'enclaves », à forte ingérence exogène. Leur cohérence et l'effet d'entraînement sur les régions d'implantation devront être mesurés. Ainsi, une nouvelle route reliant directement Inhambane et Vilanculos à Pafuri en Afrique du Sud est envisagée ; elle parait conforter la logique de 
corridor est-ouest liée à des intérêts extérieurs (en l'occurrence touristiques). Dans l'archipel de Bazaruto, la réalité écotouristique a aussi été dénoncée pour ses pressions sur la biodiversité (surpêche, rejets des déchets des hôtels) et ses côtés paternalistes (participation à la marge des communautés, acculturation). L'aménagement du site de Quirimbas entend d'ailleurs s'en éloigner (Marchés tropicaux, 2004). D'autres voix s'élèvent encore contre les ventes informelles de terrain et la mainmise étrangère (notamment de Sud-africains) sur des tronçons de plages privatisés (alors que la terre reste a priori du domaine de l'Etat, un legs de l'époque socialiste $\left.{ }^{16}\right)$. Enfin, on peut s'interroger sur les velléités de développement durable quand, sans vision globale apparente, les espaces protégés (archipels et réserves naturelles) s'ouvrent à un tourisme de luxe, souvent élitiste, entrecoupés de vastes projets d'extraction dunaire, et conduisent à des déplacements de population. Les exemples de la réserve spéciale de Maputo ${ }^{17}$ au sud de la ville, de Bazaruto et de Moatize l'illustrent (Country report, 2007 ; IAM, 2005 ; SAPA, 2007).

Dans le reste du pays, notamment dans l'hinterland, le retard en développement reste très grand. L'élaboration d'un nouveau plan d'action pour la réduction de la pauvreté absolue (PARPA II), depuis fin 2006, a bien identifié quelques priorités : outre celle de développer le secteur privé, il entend accroitre le développement rural par l'irrigation et l'établissement de micro-crédits. L'objectif est de ramener le taux de pauvreté à $49 \%$ en 2009 en privilégiant également l'éducation et la santé. Les initiatives ponctuelles, comme les "villages du Millénaire", ne doivent toutefois pas faire écran aux conditions réelles dans lesquelles réside la masse humaine adjacente ${ }^{18}$. La pauvreté rurale n'est pas seulement liée à une mise en culture insuffisante des terres. Elle découle surtout des techniques agricoles rudimentaires (absence de matériels, d'engrais). Les cultures de rente, au potentiel indéniable - le sucre grâce aux investissements sud-africains et mauriciens, le tabac qui bénéficie de la main d'œuvre zimbabwéenne ayant quitté un pays mortifié -, peuvent apporter une plus-value économique. Toutefois, vu l'augmentation mondiale du prix de produits alimentaires et les effets du réchauffement climatique sur la production de maïs dans le sud de l'Afrique (Brown, Funk, 2008), on peut être inquiet pour la consommation vivrière future. Les biocarburants, vers lesquels souhaitent s'orienter le pays, risquent également de détourner certaines terres de leur finalité nourricière. Tout cet espace demeure enfin mal desservi et intégré. Parmi les avancées, notons qu'un pont de 2,4 km est enfin érigé à Caia sur le Zambèze. Prévu pour fin 2009, il est financé par l'Union européenne, la Suède, l'Italie, le Japon et le Mozambique.

$\mathrm{Au}$ final, les défis restent de taille. La pauvreté est généralisée sur une large partie du territoire. Le marché intérieur reste peu tourné vers la consommation courante et verse dans l'économie informelle. Il s'avère que les inégalités sociales se sont en fait creusées ces dernières années, entre une minorité de nantis peuplant les beaux quartiers de Maputo et une majorité humaine restant à la traîne. Certes, le panorama est loin d'être négatif : l'IDH du pays est passé de 0,366 en 2000 à 0,428 en 2004 ; surtout, un groupe de 19 donneurs affecte dorénavant une partie de l'aide directement au budget de l'Etat et en ventile une autre dans des secteurs spécifiques. Mais la forte croissance nationale apparaitt fragile, soumise aux aléas climatiques. Concentrée sur des matières premières (lingots d'aluminium, gaz, crevettes) et les paysages (tourisme), elle reste tributaire de l'étranger, notamment des flux commerciaux. Plus largement, ce type de modèle économique, axé sur des mégaprojets à forte intensité capitalistique, pose un problème de durabilité. La création d'emplois est peu favorisée, les entreprises 
locales, en amont comme en aval, y sont peu intégrées alors que les exonérations ne dégagent guère de recettes fiscales (BafD/OCDE, 2006). Quant aux implantations d'investisseurs plus diversifiés dans les zones dites « de croissance rapide ${ }^{19}$ (province de Niassa, vallée du Zambèze, îles culturelles de Mozambique et d'Ibo), elles ne sont pas exemptes de contraintes pour plusieurs raisons: cherté des coûts d'exploitation, procédures entrepreunariales longues, collusion politique/milieu d'affaire ou encore inadéquation des infrastructures (route, électricité ${ }^{20}$ ). Une plus grande cohérence dans la promotion industrielle doit être établie si le pays veut exploiter au mieux ses potentiels. Elle permettra d'assurer, par la même, une meilleure intégration spatiale, gage de développement dans la stabilité.

\section{Conclusion}

Le Mozambique contemporain demeure un espace assez peu traité dans la littérature universitaire. Les années de troubles des décennies précédentes en ont fait une aire d'étude pour le moins éludée. Une fois la voie de la stabilité retrouvée, ce pays est resté moins étudié par les chercheurs, privilégiant les travaux sur l'Afrique australe anglophone. Le Mozambique se redécouvre aujourd'hui à la faveur de trois aspects. Il faut tout d'abord évoquer son héritage historique. Son particularisme lusophone semble agir comme un point de singularité en Afrique australe, dans un contexte inspiré de débats sur la colonisation. De plus, le pays a connu une phase socialiste autocentrée pour finalement adhérer à un credo libéral qui reste, on l'a vu, pour le moins pragmatique et opportuniste... Il faut ensuite souligner son statut de nouveau "succès africain» auprès des bailleurs de fonds. Après des années de crise, il lui confère une certaine estime qui se matérialise, par exemple, par la pléthore de documentations économiques accessibles auprès des institutions de Bretton Woods. Enfin, en raison de sa physionomie territoriale et de sa position géographique au sudest du continent, il est admis que son développement reste lié à la totalité de l'Afrique australe ; ceci, alors que le poids sud-africain ne cesse de s'affirmer et que les velléités de responsabilité africaine et de panafricanisme économique sont portées par les tournures sémantiques de "Renaissance africaine» et de Nouveau Partenariat Economique pour l'Afrique (NEPAD) ${ }^{21}$. N'oublions pas aussi que l'année 2008 voit le lancement de la zone de libre échange d'Afrique australe.

31 A travers cet article, notre but était simplement de porter un regard sur l'état actuel d'un pays en mutation. Nous souhaitions rétablir un équilibre par rapport à la somme de connaissances disponibles sur l'Afrique du Sud. Notons que l'ombre de ce pays plane ici de façon quasi-continue. Cela se vérifie à la fois sur les bifurcations historiques comme sur l'éventail des recompositions en cours (encore que ces dernières restent aujourd'hui plus largement internationales). De manière plus générale, on saisit à quel point l'influence d'enjeux et d'acteurs extérieurs a été décisive dans la construction de ce pays ${ }^{22}$. Se reposant sur quelques lignes de force historiques, au legs toujours visible sur le terrain, l'organisation spatiale du Mozambique est actuellement en passe de se complexifier, sous le poids des évolutions néo-libérales. La cohérence sociale et territoriale de ces dernières ne manque pas de susciter quelques interrogations. Pour éviter une société et un espace fonctionnant à deux vitesses, les futurs investissements étrangers se devront d'inclure au mieux les populations locales, en termes de formation, d'emploi et de véritables politiques industrielles ou écotouristiques. Les 
potentiels de développement évidents de ce pays ne pourront s'affranchir d'une redistribution des fruits de la croissance. Cela passe aussi, inévitablement, par l'émergence d'une classe moyenne et une décentralisation politique réellement effective.

\section{BIBLIOGRAPHIE}

Anuário estatístico, cidade de Maputo 2002, 2002, INE, 77 p.

Anuário estatístico, Statistical Yearbook, Moçambique 2004, 2004, INE, 132 p.

BUCUANE A., MULDER P., 2007, Exploring Natural Resoources in Mozambique: will it be a blessing or a curse?,Discussion papers, $\mathrm{N}^{\circ}$ 54E, Direcção Nacional de Estudos e Análise de Políticas, Ministério da Planificação e Desenvolvimento, 49 p.

DANIEL J., NAIDOO V., NAIDU S., 2004, “The South Africans Have Arrived: Post-Apartheid Corporate Expansion Into Africa", The State of the Nation: 2003-2004, Cape Town, HSRC Press, p. 368-390.

Dictionnaire de géopolitique, 1995, Y. Lacoste (dir.), Flammarion, Paris, 1699 p.

"Firm pays families for their loss", 2007, Sapa, 3 décembre 2007.

FOLEY C., 2007, Mozambique: A case study in the role of the affected state in humanitarian action, Humanitarian Policy Group (HGP), Overseas Development Institute (ODI), 36 p.

FOLIO F., 2007, "La criminalité à Maputo, Mozambique : origine, distribution et répercussions spatiales", Cybergeo, Espace, société, territoire, article 380.

GERVAIS-LAMBONY P., 1997, L'Afrique du Sud et les Etats voisins, Armand Colin/Masson, Paris, $253 \mathrm{p}$.

GUEBOURG J-L., BRUNET R., 1997, “Le Mozambique à reconstruire”, Mappemonde 1/97, p. 29-33.

GIRAUT F., GUYOT S., HOUSSAY-HOLZSCHUCH M., 2004, "Les aires protégées dans les recompositions territoriales africaines”, L'Information Géographique, vol. 68, décembre, p. 340-368.

HANLON J., 2004, "Do Donors Promote Corruption?: the case of Mozambique", Third World Quarterly, Vol. 25, $\mathrm{n}^{\circ}$ 4, p. 747-763.

HARRISON, G., 1999, “Corruption as 'Boundary Politics': the State, Democratisation and Mozambique’s Unstable Liberalisation”, Third World Quarterly, Vol. 20, n 3, p. 537-550.

Human Development Report 2005, 2007, PNUD en partenariat avec le SARDC, l'Information Science Research Institute (ISRI) et en collaboration avec l'INE.

JOUANNEAU D., 1995, Le Mozambique, Karthala, 224 p.

LACHARTRE B., 2000, Enjeux urbains au Mozambique, de Lourenço Marques à Maputo, Paris, éditions Karthala, $317 \mathrm{p}$. 
Le dictionnaire historique et géopolitique du XX ${ }^{\mathrm{e}}$ siècle, 2005, S. Cordellier (dir.), La Découverte, Paris, 831 p.

“Le parc des Quirimbas vers sa modernisation”, 2004, Marchés tropicaux, 2 septembre 2004.

LUNDIN, I., B., 2000, “Africa Watch: Will Mozambique Remain a Sucess Story?”, African Security Review, vol. 9, $n^{\circ}$ 3, CEEI-ISRI, Maputo.

"Maputo Special Reserve to double in size", 2005, IAM, n² 297, 26 avril 2005.

BROWN M., E., FUNK C., C., 2008, “Food Security Under Climate Change”, Science, vol. 319, $\mathrm{n}^{\circ} 5863$, p. 580-581.

Moçambique: Vida e Historia em Psikhelekedana, 2003, F. Ribeiro (coord.), Exposiçao, Centro Cultural Franco-Moçambicano, Maputo, 36 p.

“Mozambique's ports process over 75200 tons of cargo to neighboring countries", 2007, Open Society Initiative for Southern Africa (OSISA), 9 décembre 2007.

Mozambique Country Profile, 2005, The Economic Intelligence Unit Limited, 73 p.

Mozambique Country Report, 2007, The Economic Intelligence Unit Limited, février 2007.

Mozambique, 2002, BafD/OCDE, perspectives économiques en Afrique, 16 p.

Mozambique, 2006, BafD/OCDE, perspectives économiques en Afrique, 16 p.

Mozambique, 2007, BafD/OCDE, perspectives économiques en Afrique, 16 p.

Mozambique file, 2003, n 327, A Mozambique News Agency Monthly, 24 p.

PELLISIER R., 1987, « Angola, Mozambique : des guerres interminables et leurs facteurs internes », Hérodote, $n^{\circ} 46,4^{\text {e }}$ trimestre 1987, p. 83-107

"Port of Maputo expects continued rises in throughput as logistics improve", 2006, Engineeringnews, 25 août 2006.

POUSSIN S. et A., 2004, Africa Trek, Editions Robert Laffont, S.A., Paris, 436 p.

SERRA C., 2003, Em cima de uma lâmina, Um estudo sobre precaridade social em três cidades de Moçambique, Maputo, Impressa Universitária, 94 p.

"Transfrontier conservation areas : an African dream", 2003, The Journal of Southern African Tourism, vol 2, n 1, p. 86-89.

“UNDP administrator visits Millennium Village”, 2007, IAM, n 348, 17 octobre 2007.

Vitimização em Moçambique 2002/2003, Relatório Final, 2003, INE, Inquèrito aos agregados familiares sobre orçamento familiar (IAF), $31 \mathrm{p}$.

Banque mondiale Mozambique, 2007 : http://www.worldbank.org/mozambique

Companhia de Portos e Caminhos de Ferro de Moçambique (CFM) : http://www.cfmnet.co.mz/

Corridor sands : http://www.miningweekly.com/article.php?a_id=107208 et http://

sustainability.bhpbilliton.com/2005/docs/repository/aboutReport/BHPBillitonLocationsMap.pdf pour la localisation des projets mondiaux de BHP Billiton.

Estatisticas Sociais, Demograficas, Economicas de Moçambique (ESDM) : http://www.ine.gov.mz INE, Resultados Preliminares do III Recenciamento Geral da População e Habitação, Censo 2007 : http://www.ine.gov.mz/censo2007

Moatize Coal project : http://www.miningweekly.com/article.php?a_id=105256 
Moma Titanium : http://www.kenmareresources.com/moma/agreements.asp

Mozal Aluminium : http://bhpbilliton.com/bb/ourBusinesses/aluminium/mozal.jsp

Sasol Gas : http://w3.sasol.com/natural_gas/main/content.asp

Transparency international : http://www.transparency.org/

\section{NOTES}

1. Les phases historiques se sont succédées comme autant de revirements idéologiques en laissant leurs traces : période précoloniale et coloniale portugaise, caractérisée notamment par l'Estado novo de l'ère salazariste en 1933 ; ère socialiste d'austérité après l'indépendance acquise en 1975 ; années d'instabilité durant la guerre civile à ingérence étrangère, l'abandon de la doctrine marxiste-léniniste et les temps de rigueur économique dans les années 80 ; enfin, phase démocratique actuelle, à visée néo-libérale, sur fond de pauvreté chronique.

2. En 2005 , l'encours de la dette extérieure du pays se chiffrait à 4,7 milliards USD, concernant à $54,5 \%$ des créanciers multilatéraux. Un allègement de 1,3 milliards USD a été consenti par la Banque mondiale, dés 2006.

3. Lancée en 1997, à $17 \mathrm{~km}$ du centre-ville dans la zone de Matola, la fonderie d'Aluminium Mozal constitue l'un des plus gros complexes industriels en opération en Afrique australe. Elle concentre des capitaux australo-britanniques via la compagnie Billiton, mais aussi japonais et mozambicains. Mozal jouit de quelques avantages comparatifs, tels qu'une énergie électrique et une main d'œuvre peu chères. Elle se sert de son établissement portuaire mozambicain à la fois pour ses importations de matières premières et ses exportations sur le marché international (Rotterdam agissant en tant que plate-forme de transbordement de son aluminium). D'une capacité de production de 250000 tonnes lors de sa mise en opération, l'usine peut produire 506000 tonnes d'aluminium depuis 2003 (Mozal-2). L'entreprise contribue à 2,1\% du PIB mozambicain en 2002 (Mozambique file, 2003).

4. Les mégaprojets occupent une place prépondérante dans les échanges mozambicains (notamment les trois-quarts des exportations), l'aluminium et le gaz naturel arrivant loin devant les poissons/crustacés ou le coton. La part de l'industrie dans le PIB national est ainsi passée de 16 à $26 \%$ entre 1999 et 2005 du fait prioritairement de ces initiatives (l'agriculture et la pêche comptant pour $20 \%$ du PIB, les services pour $54 \%$ - BafD/OCDE, 2007).

5. La ligne du Rand fut achevée en 1894 (soit après les découvertes aurifères) : elle a initialement été financée par des capitaux allemands en tant qu'axe de contournement pour les républiques boers des hautes terres. Les mines du Transvaal ont beaucoup profité de cet axe et du port laurentin. Construit en 1899, le chemin de fer de Beira fut pour sa part lié au cuivre de Rhodésie du Nord. Harare dépend aussi fortement aujourd'hui d'un oléoduc adjacent. La ligne NacalaNyassaland reste plus récente (années 1950), davantage liée à des motifs géopolitiques.

6. Sur les 75200 tonnes de marchandises transitées en provenance et en direction des pays voisins au premier quart de l'année 2007 (OSISA), l'Afrique du Sud arrive en tête avec $49 \%$ (charbon, ferrochrome, céréale, sucre depuis Maputo), suivie du Zimbabwe avec $30 \%$ (engrais, granite, carburant, céréales à la fois depuis Maputo et Beira) et du Malawi (16\%: sucre, tabac, engrais, carburant depuis Nacala); la Zambie et le Swaziland suivent.

7. Pour les premiers, il s'agit de disposer d'un débouché et d'une force de travail à peu de frais. Pour les seconds, le traitement des salaires, par puissance interposée, s'opère pour une partie uniquement localement et pour le reste à plus long terme via la puissance tutélaire. Concrètement, l'Afrique du Sud verse en or le reliquat au Portugal et ce dernier répercute le 
salaire restant à ses travailleurs et à leur famille dans la monnaie locale (escudos). Aussi s'octroiet-il au passage une confortable marge grâce à un taux de change avantageux.

8. La relation de dépendance de la main d'œuvre du sud du Mozambique envers l'Afrique du Sud existe donc depuis longtemps. Ce tropisme est toujours actif de nos jours, bien qu'il concerne davantage les migrants clandestins. Les statistiques officielles recensaient en 200575000 travailleurs mozambicains enregistrés en Afrique du Sud, dont 60000 dans les seules mines, dans le cadre du travail migrant. Toutefois, ces chiffres occultent les migrations plus récentes dans le domaine agricole ou du bâtiment. Ce serait ainsi plus d'un million de Mozambicains qui à cette date travaillent légalement ou illégalement en Afrique du Sud. Durant les trois premiers trimestres de l'année 2001, 61000 d'entre eux ont été expulsés (Mozambique Country Profile, 2005).

9. Ce dernier, dans un contexte de guerre froide, percevant d'un mauvais oeil ces idéologies socialistes et les relations intimes tissées avec l'URSS et Cuba, aux portes mêmes d'un régime capitaliste et nationaliste.

10. Un des legs de ce conflit étant aujourd'hui les mines anti-personnelles présentes sur le terrain, ainsi que les caches d'armes qui contribuent à la hausse sensible des crimes violents à Maputo et à Beira (Folio, 2007).

11. Pour être précis, le Departamento Nacional da Estatistica (DNE) soulignait, à l'année 1991, que cette accélération de l'urbanisation avait particulièrement concerné les deux centres urbains de Maputo et Beira. Pauvreté et instabilité ont évidemment précipité les masses humaines dans ou autour des deux principales agglomérations côtières du pays, en quête de sécurité physique/ alimentaire et d'une recherche d'anonymat.

12. Celle-ci doit composer avec l'éloignement naturel de ses ports du Gauteng. Elle a aussi pour souci de réguler leur problème de congestion, port de Durban en tête (dans l'attente de celui de Ngqura au Cap de l'Est).

13. D'une superficie de $35000 \mathrm{~km}^{2}$, le parc épouse les anciens circuits de migration des animaux et ambitionne de devenir un pôle du tourisme en Afrique sub-saharienne (The Journal of Southern African Tourism, 2003). Cette réserve élargie, supportée par la Peace Park Foundation, le World Wild Fund (WWF) et les Etats respectifs, s'inscrit, depuis 2006, dans un schéma d'aménagement à la mode en Afrique australe. Elle suit un triple objectif qui sous-tend le tourisme durable : la promotion de la paix par la collaboration entre les nations, la conservation de la biodiversité et le développement par l'implication des communautés. Outils d'expansion de l'influence sudafricaine, ces espaces entérinent aussi une certaine sanctuarisation (Giraut, Guyot, HoussayHolzschuch, 2004).

14. Par exemple, le taux d'alphabétisation, s'il est de $34 \%$ dans les campagnes, atteint $70 \%$ en milieu urbain (qui abrite $36 \%$ de la population totale en 2007, INE). La mortalité infantile est de $143 / 1000$ dans les villes contre 192/1 000 en zone rurale. Pour finir, l'accès à l'eau potable est de $27 \%$ dans les campagnes contre $64 \%$ en ville.

15. Dans les parcs nationaux créés, certaines des îles hébergent une panoplie d'ecolodges intégrés dans la nature. Elles ont adopté la voie de l'écotourisme en faisant participer les pêcheurs locaux : taxe hôtelière et pourcentage du droit d'entrée redistribués, vente de poissons, gestion des sites de camping...

16. Entretien avec M. Aristides Baloi, département de géographie de l'Université Eduardo Mondlane.

17. Passée de 800 à $1600 \mathrm{~km}^{2}$ depuis 2005, la réserve spéciale d'éléphants de Maputo est un secteur riche en biodiversité (diversité ornithologique, zone de ponte des tortues sur la côte). Les autorités veulent en faire un pôle de l'écotourisme transnational (avec l'Afrique du Sud et le Swaziland). Elles doivent toutefois résoudre un conflit qui les opposent aux fermiers concernant la circulation des pachydermes (d'où l'objectif de la clôture du Futti corridor), ainsi que régler la question des compensations attribuées aux communautés rurales déplacées. 
18. Promu par le PNUD, les villages du millénaire s'inspirent de projets pilotes menés en Ethiopie et au Kenya. Ils visent à extirper les habitants de la pauvreté sur des périmètres restreints, avec l'aide des donneurs (IAM, 2007). Au Mozambique, pour le moment, seul le village de Chibuto (à Gaza) a été retenu. Il en est prévu onze au total (un par province), chacun accueillant 5000 habitants, pour un investissement annuel total de 3,3 millions USD.

19. Les investissements y sont exemptés de droits sur les transferts de titres immobiliers, de droits sur l'importation de certains biens et bénéficient d'un crédit d'impôt à hauteur de $1 / 5^{\mathrm{e}}$ de l'investissement total.

20. Précisons néanmoins que depuis l'accord de novembre 2005, le Mozambique a effectué un premier versement pour acquérir $67 \%$ de l'usine hydraulique de Cahora Bassa, qui appartenait encore au Portugal (et avec qui le pays conservait un litige). Avec une participation de l'Etat qui s'élève dorénavant à $85 \%$ (et un arriéré de 700 millions USD), les autorités espèrent engager de nouveaux investissements dans la capacité de production électrique, avec l'appui d'entreprises privées. L'électricité est devenu le troisième poste d'exportation du pays.

21. Processus innovant sur le papier par son caractère partenarial africain (et où le poids de l'Afrique du Sud y est notable), le NEPAD entend définir ses propres politiques de développement dans un souci de bonne gouvernance. Les priorités identifiées jusqu'à présent ont surtout porté sur les infrastructures et la sécurité alimentaire, des aspects clés pour le Mozambique. Au nombre des quelques initiatives qui ont ici vu le jour, citons des actions conjointes dans le domaine de la pêche ou le NEPAD e-Schools dans l'éducation. Des négociations ont aussi été entamées concernant le pont sur la Rovuma entre le Mozambique et la Tanzanie.

22. On peut rapidement les énumérer : présence arabe côtière, prazos portugais, compagnies concessionnaires privées, intérêt britannique dans l'hinterland, colonisation salazariste, mouvement de libération d'inspiration tanzanienne, régime socialiste dans un contexte de guerre froide, rebelles instrumentalisés par l'Afrique du Sud, politique d'austérité guidée par le FMI, aide internationale, investissements étrangers, notamment sud-africains.

\section{RÉSUMÉS}

Cet article esquisse un état des lieux géographique de la république du Mozambique. L'organisation spatiale de ce pays s'inscrit à la fois dans des mutations en cours et dans un héritage complexe. Une approche multiscalaire permet d'en rendre compte tout en recourant à la dimension géohistorique. L'influence d'enjeux et d'acteurs extérieurs sur l'aménagement de cet espace est prégnante. Les réalités spatiales et les logiques socio-économiques en cours peuvent en témoigner, que ce soit la forme singulière de ce pays ou encore le hiatus croissance/ développement observé sur place. Les lignes de force originales, inscrites sur l'espace mozambicain, associent en fait des "pénétrantes" transnationales couplées à une excentration historique de sa première ville et de nouveaux pré-carrés dynamiques, inscrits dans la mondialisation, dont il faudra questionner la cohérence sociale et territoriale.

The aim of this article is to draw out the geographical broad lines of the republic of Mozambique. The country's spatial organisation is part of the present mutations as well as its complex heritages. Different scales will be considered. Moreover, we will point out both the historical and geographical background. The paper will try to demonstrate that lots of outer stakes as well as external actors have played a major role on the spatial reality of Mozambique. The 
contemporaneous spatial and socio-economical realities can attest to this point, such as the specific physiognomy of this territory or the gap between national growth and social development. Finally, the spatial Mozambican singularities display several internal "corridors" connected to foreign countries, the localisation of the capital-city, Maputo, at the extreme south periphery of the country and new strategic areas linked to globalisation. The social and spatial coherence of the latter will be issued.

\section{INDEX}

Mots-clés : développement., dynamique historique, géohistoire, influence exogène, Maputo, mondialisation, Mozambique, organisation spatiale, recomposition

Keywords : external stake, globalisation, historical dynamic, Maputo, Mozambique, social development., spatial mutation, spatial organisation

\section{AUTEUR}

\section{FABRICE FOLIO}

Fabrice Folio (fabrice-jm.folio@univ-reunion.fr) est enseignant-chercheur en géographie à l'Université de La Réunion, rattaché au Centre de Recherches et d'Etudes en Géographie de l'Université de La Réunion (CREGUR). Ses activités de recherche portent prioritairement sur la territorialisation et les dynamiques d'acteurs, héritées et en mutation, au sein des espaces urbano-industriels et touristico-résidentiels d'Afrique australe et orientale (littoral de l'océan Indien : Afrique du Sud, Mozambique, Tanzanie). Il a notamment publié :

- « La criminalité à Maputo, Mozambique : origine, distribution et répercussions spatiales », Cybergeo, 2007, Espace, Société, Territoire, article 380.

"Construction géohistorique du KwaZulu-Natal (Afrique du Sud). Une terre de conflits et d'enjeux à l'origine du présent cadre spatial », EspacesTemps.net, 2007, Textuel.

- « Les condominios à Maputo : enjeux sociétaux et spatiaux autour de l'implantation des ensembles résidentiels sécurisés dans la capitale mozambicaine » in Les annales de géographie, 2007, p. 247-270. 